\title{
The impact of street characteristics on older pedestrians' perceived safety in Shanghai, China
}

\author{
Hao Wu \\ Tongji University \\ 2015_wh@tongji.edu.cn \\ Yong Chen (corresponding author) \\ Tongji University \\ ch_yong2011@tongji.edu.cn
}

\author{
Zhaoxi Zhang \\ Aarhus University and \\ Tongji University \\ zhangzx@envs.au.dk \\ Junfeng Jiao \\ The University of Texas at Austin \\ jjiao@austin.utexas.edu
}

\begin{abstract}
Land-use patterns and rapid urban sprawl greatly influence older adults' mobility in China. Older pedestrians' safety issues are crucial because these people are more frequently injured in traffic accidents. This research aims to investigate what street characteristics influence perceived safety among older pedestrians in Shanghai, China. A mix of research methods containing both quantitative and qualitative analyses were employed. The researchers recruited 68 elderly urban dwellers who each agreed to take a perceived safety survey using 39 simulated streetscape images that contained 12 street characteristics extracted from four sectional zones of streets. Ordinal logit regression was performed to investigate the impact of street characteristics on older pedestrians' perceived safety. A semi-structured, in-depth interview was conducted with 8 out of the 68 participants. It was found that the street interface type, elevation differences, footpath width, paver directions, isolation facility type, and vehicle traffic all affected pedestrians' perceived safety after adjusting for individual demographic attributes and the physical limitations of the participants. Understanding the street characteristics related to perceived safety will contribute to making more inclusive and walkable cities.
\end{abstract}

Keywords: Perceived safety, simulated streetscape, built environment, older pedestrians, functional decline

\section{Article history:}

Received: April 15, 2019

Received in revised form: April

20, 2020

Accepted: May 1, 2020

Available online: November 10, 2020

Copyright $2020 \mathrm{Hao}$ Wu, Zhaoxi Zhang, Yong Chen, \& Junfeng Jiao http://dx.doi.org/10.5198/jtlu.2020.1588

ISSN: 1938-7849 | Licensed under the Creative Commons Attribution - Noncommercial License 4.0

The Journal of Transport and Land Use is the official journal of the World Society for Transport and Land Use (WSTLUR) and is published and sponsored by the University of Minnesota Center for Transportation Studies. 


\section{Introduction}

Promoting walking is important from both an environmental and public health perspective. It as a means of transport reduces air pollution by taking cars off the road, while also has myriad health benefits such as inducing sufficient physical activities to maintain individual health status (Chaudhury, Campo, Michael, \& Mahmood, 2016). These benefits are particularly significant for older adults who stand to benefit the most from exercise and who are most vulnerable to air pollution (Shumake, Sacks, Lee, \& Johns, 2013). Thus, making older adults' walking activities easy and safe should be a key public policy concern for planners, policymakers, and government officials. As of 2018, the number of senior citizens (age> 65) in China reached 167 million (National Bureau of Statistics of China, 2019). In Shanghai, the older adults group (age> 60) accounts for 33.3\% of the registered population (Bureau of Statistics of Shanghai, 2018). Pedestrian's safety issues are distinctively important among older adults as they are more likely to be involved in traffic accidents and the fatality and disability rates in accidents are much higher than those of other ages (Zhang, Xiang, Jing, \& Tu, 2011). Previous survey found that walking was the most prevalent travel mode among older adults and the average outdoor walking travel time of older adults (>60) in Shanghai was twice more compared to those of younger citizens (Chen, Jiao, Mao, $\& \mathrm{Wu}, 2017)$. This dominant share in travel modes is particularly prevalent in most Chinese contexts due to the decreased individual mobility as well as the extremely low rate of driver's license possession of elders (Feng, 2017). However, rapid urban sprawl has changed land-use patterns in Chinese cities (Yang \& Gakenheimer, 2007) and as a result, has made walking, formerly a practical means of transportation or recreation, more difficult. What is more, the impacts that change in urban land use brings on older pedestrians' walking activities are intensified due to the prevalence of large automobile traffic volume and pedestrian crowdedness in highly-populated metropolitans, like Shanghai. While much attention has been paid to issues associated with aging population of China, research on elder's walking safety issues from street built environment perspective is limited. A better understanding of the relationship between street characteristics and walking safety will contribute to making cities more inclusive and improve the quality of life (QoL) of the elderly. Via a novel survey method, the current research aims to investigate the impacts of street characteristics on older pedestrians' perceived safety.

\section{$2 \quad$ Literature review}

\subsection{Perceived safety}

The term "perceived safety" in the current research particularly indicates the perception related to safety that one perceive when he or she walks on the street, including safety from crime and safety of traffic. Perception is the process of attaining awareness or understanding of sensory information. What one perceives is a result of interplays between past experiences, one's culture and the interpretation of the perceived (Ewing \& Handy, 2009). According to affordance theory (Gibson, 1977), individual decision of making a specific behavior is highly dependent on the perceived affordance of the environment which supports the forthcoming behavior. In Maslow's theory of hierarchy of needs (Maslow, 1943), safety is one of the five needs which influence individual behaviors. A higher level of behavior need will not be motivated if the lower level need has not been satisfied. As for walking behavior, the theory of walking needs introduced a hierarchy consisted of feasibility, accessibility, safety, comfort, and pleasurability needs that are considered within the walking decision-making process. Within this hierarchical structure, if the walking safety need cannot be met, a person would not consider his or her need for comfort or pleasurabilty when deciding whether to walk (Alfonzo, 2005). Research found that for each increase 
in perceived safety from crime on a five-point Likert scale, a 7.0 minute per week increase in residents' recreational habits was occurred (Foster et al., 2016). Older adults would be more active if they had easy access to a safe path within neighborhoods (Booth, Owen, Bauman, Clavisi, \& Leslie, 2000).

\subsection{Correlates of older adults' walking activities}

From an ecological perspective, individual behaviors such as walking activities are influenced by factors from individual and environmental level (Li, Fisher, \& Brownson, 2005; Loukaitou-Sideris, 2006; Sallis et al., 2006). Previous literature found individual age was particularly predominant in the fear of crime research which indicated that the correlation magnitude between perception of safety and the frequency of walking the streets alone at night were stronger in the older group(over 65s) than in the youth group (under 25s) (Tulloch, 2000). Inter-personal factors such as participation in friends and families were tested to be positively associated with more physical activities in elder groups (Booth et al., 2000). The impacts of built environment on older pedestrians' perceived safety are embodied in both neighborhood level and street level. As people age, older adults' daily activities are basically constrained to their immediate neighborhood (Chaudhury et al., 2016). Neighborhood residential density, commercial density, street connectivity, and land-use mix were regarded as motivators and widely used to evaluate how walkable a neighborhood was (Frank et al., 2010). To the contrary, incivilities within neighborhood (e.g., deteriorated or abandoned buildings, litter, graffiti, etc.) were responsible for generating perceptions of risk and fear which restrained walking activities (Loukaitou-Sideris, 2006). A higher proportion of retail and park facilities within neighborhoods invited more walking activities (Foster, Giles-Corti, \& Knuiman, 2010), supporting the assertion that "eyes on the street" enhanced perceptions of safety (Jacobs, 1961). For traffic safety, research also found a well-connected road network with higher intersection counts per area was proved associated with fewer pedestrian crashes (Marshall \& Garrick, 2011).

As for what older adults feared most when walking on the street, research revealed that most older pedestrians had a fear of being attacked, collisions and/or falls (Burton \& Mitchell, 2006). This research also proposed inclusive street design principles which called for six major requirements for outdoor environments to be age-friendly: familiar, legible, distinctive, accessible, comfortable and safe. On the street level, both lighting and surveillance factors were found related to the perception of walking safety (Pikora, Giles-Corti, Bull, Jamrozik, \& Donovan, 2003). Considering the decreased mobility, research found ramps or slopes, rather than steps, were more preferred among older pedestrians especially when the sidewalk had altitude differences (Burton \& Mitchell, 2006). Zamora, Alcántara, Artacho, and Cloquell (2008) investigated the influence of pavement design features in older pedestrians' safety perception and found pavement textures and pavement joints were the most influential factors. Streets being longer, better connected, absent of steps and equipped with attractive views were more likely to be selected by older pedestrians when they participated in recreational walking activities (Joseph \& Zimring, 2007). Borst et al. (2009) also found that street characteristics like interface type along the street, pavement maintenance, and roadside amenities were correlated with the likelihood of a segment being chosen by elders.

\subsection{Measure of perceived safety}

While revealed preference $(\mathrm{RP})$ method was conventionally employed to investigate the relationship between travel behavior (i.e., frequency, duration, collision, etc.) and built environment characteristics (Wardman, 1988), perceived safety research relies more on statements or views acquired through psychometrical measurement in individual level. Additionally, measuring the attributes of streets related to perceived safety was an issue of challenge because of the complicity of real-world streets which consisted 
of numerous features. So the stated preference (SP) method is particularly appropriate for perceived safety research. Streetscape, either in static (Van Cauwenberg et al., 2014) or in dynamic form (Ewing \& Handy, 2009; Kang, Xiong, \& Mannering, 2013), visually addressed the gap between built environment and individual perceptions on certain qualities related to walkability. For instance, Van Cauwenberg et al. (2014) employed a set of manipulated real-world streetscapes to investigate the attractions of street built environment on older pedestrians' commuting trips. Ewing and Handy (2009) employed streetscape video clips to measure the quality of walkable cities. Recently, the human-scale approach employed emerging technologies to better investigate the impact of street built environment on pedestrians behaviors and emic experiences (Li et al., 2015; Ye, Richards, Lu, Song, Zhuang, Zeng, \& Zhong, 2019). Ye et al. (2019) quantified the pedestrians' daily exposure to eye-level street greenery by integrating high resolution measurements on both greenery and segment accessibility. Notably, most existing studies on older pedestrians scarcely controlled for the effects of age-related body functional declines when investigated the relationship between individual perceptions and built environment characteristics, judging elder groups by physiological age only, even though most older adults who suffered from age-related functional declines, such as sensory or cognitive declines, had inconsistent perceptions of environment features compared to the younger people. For instance, researchers observed inconsistencies between what older adults perceived and what urban environments objectively equipped with (Cao, Mokhtarian, \& Handy, 2010; Michael, Beard, Choi, Farquhar, \& Carlson, 2006). And older pedestrians were less sensitive to complicated urban settings than youngers do due to the decreased vision depth with age (Burton \& Mitchell, 2006).

The conventional streetscape method employed in previous research either relied on real-world streetscape which inevitably contained interferential factors like weather condition and temporal factors (Traunmueller, Marshall, \& Capra, 2016; Van Cauwenberg et al., 2014), or employed virtual streetscape maps from bird's-eye view (Harvey, Aultman-Hall, Hurley, \& Troy, 2015) which ignored emic pedestrians' perceptions. Simulated streetscape images from pedestrians' views are probably a pathway of high-efficiency to investigate perceived safety issues of older pedestrians because it not only mitigate the distractions from real-world street images but also acquire more accurate perception information from pedestrians' eye-level. The current research explored a novel streetscape image set using simulated street scenes to measure older pedestrians' perceived safety and to investigate street built environment characteristics related to perceived safety of older pedestrians in Shanghai, China, controlling for individual health status.

\section{$3 \quad$ Research design}

A mixed approach containing both quantitative and qualitative research methods was employed in the current research. Based on two surveys, measurements of older pedestrians' perceived safety and their individual-level factors were acquired. A multivariate regression model was used to investigate the relationship between perceived safety and street characteristics, as well as individual level factors such as demographical and age-related body functional variables. Subsequently, a semi-structured interview was conducted through telephone calls to obtain the in-depth descriptions and cognitions of safety-related characteristics of the streets from the participants. Participants' statements extracted from the interviews were used as supplementary information to better understand the results of quantitative analysis. The research design framework was shown in Figure 1. 


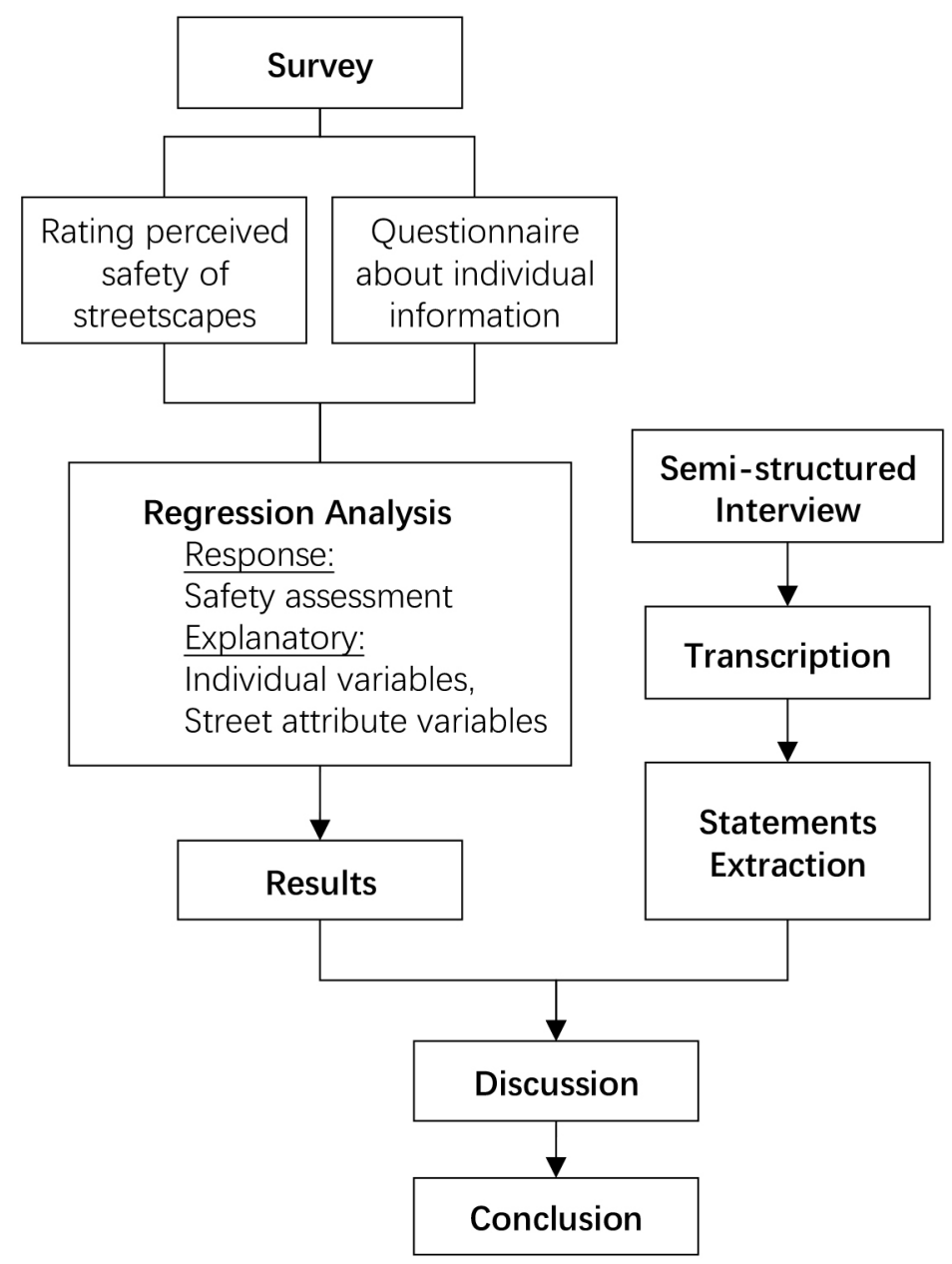

Figure 1. Research design framework

\subsection{Survey protocol}

Considering the age-related sensory and cognitive declines of older adults, the current research adopted a set of simulated streetscape pictures which only contained several street environmental characteristics. The merits of this protocol were that older adults could focus more, compared to real-world streetscape pictures, on safety perception survey since other confounding factors like weather conditions were not mingled in the streetscape. Due to the high population density and the vast usage of non-motor vehicles in daily life, road patterns and right-of-way in Chinese cities and primary travel mode of Chinese citizens are quite distinct from those of other countries like the United States (Chen, Jia, \& Lau, 2008; Tana \& Chai, 2015; Wu, Chen, \& Jiao, 2019). As a result, the current research identified four sectional zones differed by their locations on typical Chinese urban streets, and composed them into the simulated streetscape pictures. Particularly, building interface zone was located at the very proximate of buildings along the street, followed by sidewalk zone, isolation zone, and vehicle zone at the outermost edge of the street, which included a non-motor zone (if any) and motor zone (Figure 2). 


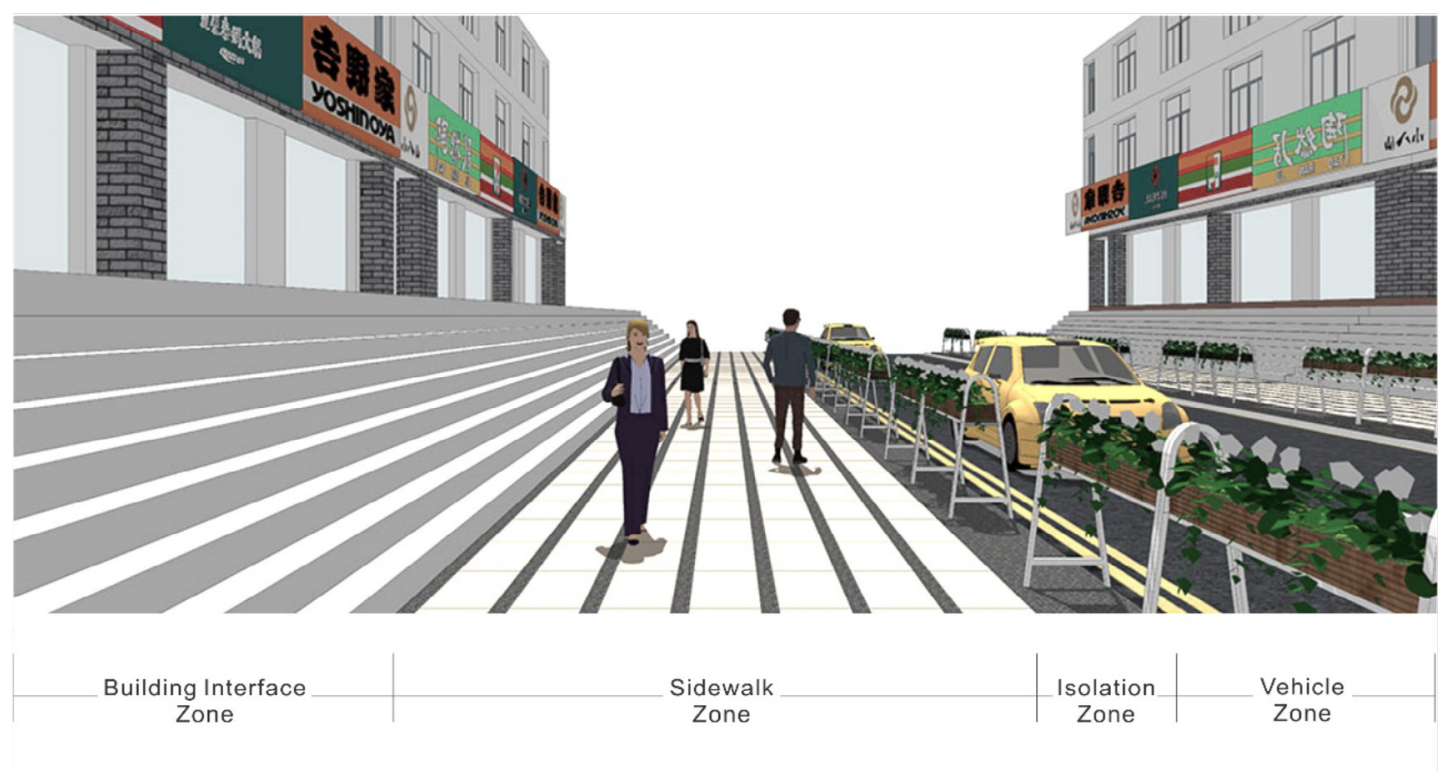

Figure 2. Street partition zones of simulated streetscape picture

Each sectional zone contained several street characteristic variables. Specifically, characteristic variables in Building interface zone were building interface type, outer setting facilities (e.g., exterior seats of street shops), and steps. The presence of urban seats and catering facility along the street were used as proxies of urban vitality both in Chinese context (Ye, Li, \& Liu, 2018) and European classic urban streets (Gehl, 2013). Characteristic variables in sidewalk zone were footpath width, pedestrian crowdedness, sidewalk maintenance, presence of tactile paving, and paver direction(texture). Compared to conventional street research targeted to the whole population, the current research provided insight into some finer sidewalk elements, such as pavement textures, which was tested crucial for older pedestrians in previous research (Zamora et al., 2008). In isolation zones, street characteristic variable particularly referred to the type of isolation facilities (i.e., handrails, raised flower beds, isolation piles, bicycle docks, etc.) which were widely used in most Chinese cities. In vehicle zone, characteristics included the presence of street parking lots, non-motor vehicle riders, and motor vehicle traffic volumes. Detailed description and measure (observed value) of each street characteristic variable are listed in Table 1. Two investigators (HW and ZZ) rated each streetscape picture's pedestrian crowdedness on a 5-point scale. Intra-class correlations were tested and results showed a good inter-rater reliability (ICC $=0.785, \mathrm{p}<0.001)$. Then the crowdedness feature of each streetscape picture was determined by the mean value of two raters' ratings, and then was re-coded into a 9-point scale with a step length of 1.0. In sum, 12 street characteristic variables were identified and they were used as explanatory variables to investigate their correlations with older pedestrians' perceived safety. 
Table 1. Measures and descriptions of street characteristic

\begin{tabular}{|c|c|c|}
\hline Street characteristics & Feature coding & Description \\
\hline Interface type & $\begin{array}{l}1=\text { wall, } 2=\text { commercials, } 3=\text { fences(ref.), } \\
4=\text { plantations }\end{array}$ & $\begin{array}{l}\text { Wall and fences are common boundaries dividing } \\
\text { private dwelling communities and public urban } \\
\text { spaces in the Chinese context. Plantations are a } \\
\text { sort of buffer zone between dwelling community } \\
\text { boundaries and sidewalks. Commercials indicate the } \\
\text { strip shops within neighborhoods. }\end{array}$ \\
\hline Outer settings & $1=$ presence, $0=$ absence $($ ref.) & $\begin{array}{l}\text { This facility, like a set of seats, was usually provided } \\
\text { by the shop owner for their own business. }\end{array}$ \\
\hline Steps & $\begin{array}{l}1=\text { none } \operatorname{steps}(\text { ref.), } 2=\text { three steps, } 3=\text { ten } \\
\text { steps }\end{array}$ & $\begin{array}{l}\text { A 3-step was minor elevation difference in front of } \\
\text { a street shop, prevent rainwater from backflow into } \\
\text { the property. A 10-step means a major elevation } \\
\text { difference linking sidewalks and the platform owned } \\
\text { by property owners. }\end{array}$ \\
\hline Footpath width & $\begin{array}{l}1=1.5 \text { meter(ref.), } 2=3.0 \text { meter, } 3=5.0 \\
\text { meter }\end{array}$ & $\begin{array}{l}\text { Footpath includes municipal sidewalk area and the } \\
\text { shop front area, the latter was private but it provides } \\
\text { spaces for pedestrians. }\end{array}$ \\
\hline Pedestrian crowdedness & $\begin{array}{l}1=\text { very low(ref.) to } 9=\text { very high, step } \\
\text { length }=1\end{array}$ & $\begin{array}{l}\text { Subjectively measured by two investigators based } \\
\text { on a comprehensive understanding of number of } \\
\text { pedestrian, the footpath width and isolation type } \\
\text { presented in each simulated picture. }\end{array}$ \\
\hline Maintenance & $\begin{array}{l}1=\text { presence of broken tiles, } 0=\text { absence } \\
\text { of broken tiles(ref.) }\end{array}$ & $\begin{array}{l}\text { This variable reflects the quality or maintenance of } \\
\text { sidewalks }\end{array}$ \\
\hline Tactile paving & $1=$ presence, $0=$ absence(ref.) & $\begin{array}{l}\text { Raised pavers were used in this facility for visually- } \\
\text { impaired individuals }\end{array}$ \\
\hline Pavers directions & $1=$ isotropic(ref.), $2=$ parallel, $3=$ vertical & $\begin{array}{l}\text { "Vertical" indicates the paver textures are vertically } \\
\text { intersected with the pedestrian flow, compared to } \\
\text { "parallel" textures. "Isotropic" indicates pavers are } \\
\text { quadrate. }\end{array}$ \\
\hline Isolation type & $\begin{array}{l}1=\text { none, } 2=\text { handrail, } 3=\text { flower bed, } \\
4=\text { pile, } 5=\text { bicycle dock, } 6=\text { bushes, } \\
7=\text { trees, } 8=\text { mixed type(ref.) }\end{array}$ & $\begin{array}{l}\text { There are seven single types of isolation facilities, } \\
\text { plus one mixed type, which was a composition of } \\
\text { two or more single types. }\end{array}$ \\
\hline Parking lot & $1=$ presence, $0=$ absence(ref.) & This facility indicates the street parking lot. \\
\hline Non-motor vehicle & $1=$ presence, $0=$ absence(ref.) & $\begin{array}{l}\text { This variable identifies whether at least one bike } \\
\text { rider was presented in the simulated streetscape } \\
\text { pictures }\end{array}$ \\
\hline Motor vehicle volume & $1=$ low, $2=$ medium, $3=$ high(ref.) & $\begin{array}{l}\text { This variable was measured by the amounts or } \\
\text { crowdedness of vehicles presented in the simulated } \\
\text { streetscape pictures. }\end{array}$ \\
\hline
\end{tabular}

(ref.) indicated this feature was regarded as a referred category in the regression model.

All possible combinations of street variables with each unique feature presented would yield thousands of simulated streetscape pictures. To embody as various features of the selected variables as possible in the simulated streetscape and to ease the burden of perceived safety survey on older adults, an orthogonal experimental design method which was able to pick up the most representative cases from a comprehensive trial (Ross, 1988), was employed to determine the final combinations for generating streetscape pictures. The orthogonal experiment computing yielded 81 initial combinations. 39 
combinations were remained in the current research while the others were dropped due to the presence of contradictory information that rarely existed in the real world. All remained combinations were then used to generate simulated streetscape pictures by SketchUp software (version 2017, Trimble Inc., Sunnyvale, CA).

\subsection{Participants}

In December 2017, 68 older residents (mean age=68.9, $S D=6.6, \min =58, \max =85$ ) who lived in Yangpu District in Shanghai participated in the survey. With the support from local community workers, recruitment posters were mounted on bulletin board of each community. Older adults who intended to take the survey voluntarily gathered at a local community facility by schedule. The result of this recruitment reflected a gender bias that female participant $(n=51)$ accounted for three fourths of the total. Although the proportion of males in Shanghai is close to $49.55 \%$ according to 2017 census data, we did accept the survey result as many studies indicated that older female residents were more active in community social activities while male residents spent more on exercise activities (Chen, 2010; Schlozman, Burns, Verba, \& Donahue, 1995).

\subsection{Survey process}

The survey contained two parts, with a rating task acquiring the perceived safety data and a questionnaire-survey acquiring individual demographics and age-related body functional declines information.

Prior to rating tasks, a trained guide (graduate students in urban design program) gave a 5-minute brief introduction to each participant, which included the survey purpose and instructions to the survey. In rating tasks, participants were instructed to assess the perceived safety of 39 simulated streetscape images one by one on a laptop. To minimum the skewed error of safety assessment by individual preference, two reference simulated pictures (pic. A and pic. B) reflecting a relatively unsafe/safe scene were also presented on the user interface of the protocol. Participants were asked to rate "How safe would you feel in this picture?" in a five-point scale. Response choices shown in the user interface were (1) "less safe as pic. A", (2) "as safe as pic. A", (3) "between pic. A and pic. B", (4) "as safe as pic. B", and (5) "safer than pic. B" (Figure 3). During the individual rating survey, the trained guide illustrated and recorded any queries raised by older participants, as well as timing the survey in case of any distractions of the participants. While the expected time consumption on each rating was 30 seconds, the actual time consumption of completing the rating tasks in most cases exceeded the expectation due to distractions. After the rating survey, participants were invited to complete a self-administrated questionnaire through which individual demographic information such as gender and age, and their body functional limitations related to walking, such as visual and hearing impairments and arthritis diseases, as well as personal contact information for further interviews were acquired. Participants were also allowed to quit if they did not expect to take the survey at any time. On average, it took $40 \mathrm{~min}$ individually to finish the whole survey process and finally 68 older participants completed the survey.

Participants' gender disproportion was noticed and then investigators performed a K-S test to verify if any differences of distribution of targeted variable (perceived safety) existed between two samples(male and female participants). No significant inconsistence of distribution of perceived safety was observed $(\mathrm{p}=0.774)$, which indicated gender, though disproportional, did not show significant impact on older pedestrians' perceived safety assessment in the current recruited samples. Considering the time and labor cost, researchers did not recruit more participants to balance the gender bias of the participants. 


\section{How safe would you feel on this picture?}
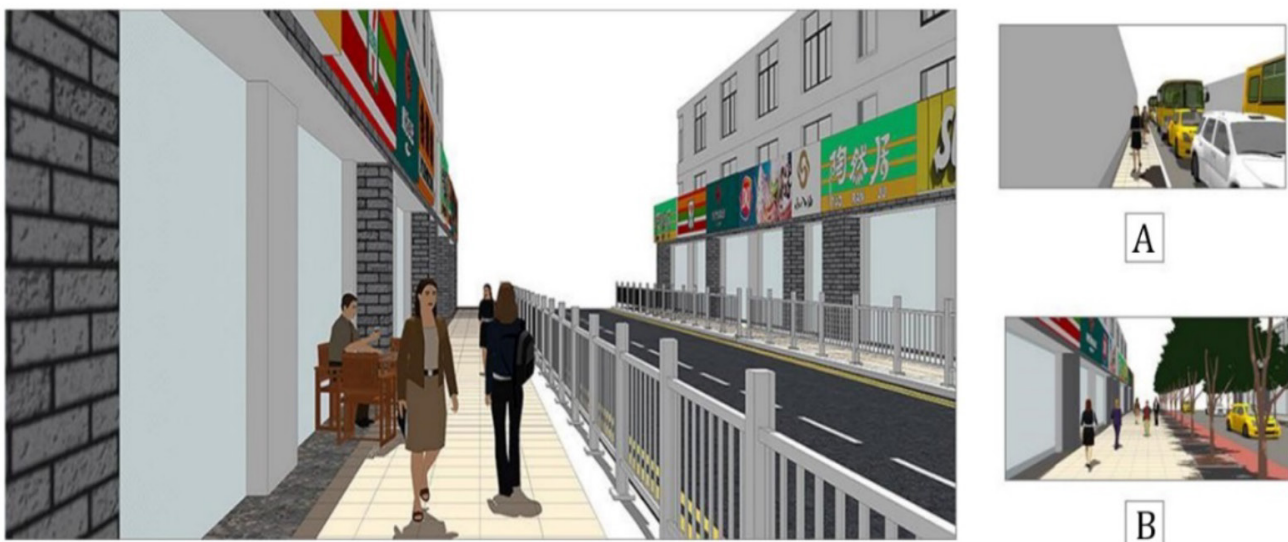

A

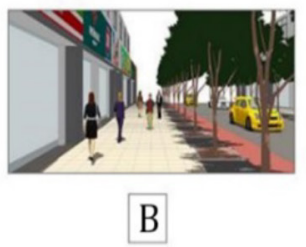

Less safe as A

As safe as A

Bewteen A, B

As safe as B

More safe than B

Figure 3. A user interface sample of rating perceived safety on a simulated streetscape

\subsection{Regression analysis}

The response variable in the research, the safety perception on a certain stimulated streetscape picture, has multiple ordered categories. Thus an ordinal logit regression analysis was performed to investigate the impact of street characteristics on older pedestrians' perceived safety, controlling for individual demographic attributes and functional limitations. A significance level of 0.05 was utilized up to determine which streetscape variables were significantly correlated with perceived safety. Both individual level and the street built environment level characteristics entered as explanatory variables the model. All re-coding and analysis were processed in Stata MP (version 14, StataCorp LLC., College Station, TX).

\subsection{Qualitative research}

\subsubsection{In-depth interview}

A semi-structured interview of the enrolled participants was conducted through telephone calls after the 2017 perceived safety survey. An interview guide consisted of 12 open-ended questions related to street built environment and pedestrian's safety issues was developed. Interviewees were initially asked, "Which kinds of environment issues decrease your safety perception when you walk on/cross a street?" Based on the responses, questions on street built environment characteristics like street shops, steps, sidewalk pavers, isolation strips, street furniture, plantations, and vehicles were proposed afterward. At last, interviewees were also asked to indicate if any built environment features related to safety have been changed since the previous perceived safety survey. 


\subsubsection{Interview participants}

In the 2017 survey, 39 out of 68 respondents stated they would like to participate in the future follow-up survey and logged their personal contact information. Investigators attempted to contact every potential interviewee by telephone in October 2019. 20 out of 39 respondents could not be reached due to either non-existent phone numbers $(\mathrm{n}=9)$ or no responses $(\mathrm{n}=11)$. After stating the aim of the telephone call, 11 respondents refused to participate in this follow-up interview. Finally, only 8 ( 4 males and 4 females) out of the 68 initial respondents completed the semi-structured interview. Oral consent for audiotaping the conversation was obtained prior to the formal interview on each participants. All audiotaped statements were transcribed for further discussion.

\section{$4 \quad$ Results}

\subsection{Descriptive analysis}

68 older adults finished the perceived safety survey in 2017, yielding 2652 rating observations (39 image ratings from each of 68 older adults) in total (Table 2).

Individual-level characteristics of the current sample were presented in Table 3. Among all participants, 17 were male and 51 were female. $16(23.53 \%)$ were aged older than 75 years. The survey on individual body functional limitations related to walking activities showed that 36 (52.94\%) out of 68 respondents were suffering from visual impairments and 31 (45.59\%) from arthritis disease. Only 10 (14.71\%) respondents reported their daily walking activities were affected by hearing impairments. 
Table 2. Descriptive results of the perceived safety survey $(\mathrm{n}=2652)$

\begin{tabular}{|c|c|c|c|c|c|c|}
\hline \multirow[b]{2}{*}{ Picture No. } & \multicolumn{6}{|c|}{ Perceived safety level } \\
\hline & $\begin{array}{r}1 \\
\text { "less safe as } \\
\text { pic. A" }\end{array}$ & $\begin{array}{r}2 \\
\text { "as safe as pic. } \\
\text { A" }\end{array}$ & $\begin{array}{r}3 \\
\text { "between pic. } \\
\text { A and B" }\end{array}$ & $\begin{array}{r}4 \\
\text { “as safe as pic. } \\
\text { B” }\end{array}$ & $\begin{array}{r}5 \\
\text { "safer than pic. } \\
\text { B" }\end{array}$ & Total \\
\hline 1 & 0 & 4 & 4 & 13 & 47 & 68 \\
\hline 2 & 0 & 0 & 42 & 9 & 17 & 68 \\
\hline 3 & 0 & 22 & 31 & 9 & 6 & 68 \\
\hline 4 & 0 & 4 & 9 & 27 & 28 & 68 \\
\hline 5 & 0 & 33 & 26 & 4 & 5 & 68 \\
\hline 6 & 0 & 0 & 37 & 23 & 8 & 68 \\
\hline 7 & 0 & 5 & 0 & 28 & 35 & 68 \\
\hline 8 & 0 & 14 & 14 & 27 & 13 & 68 \\
\hline 9 & 12 & 56 & 0 & 0 & 0 & 68 \\
\hline 10 & 0 & 12 & 16 & 8 & 32 & 68 \\
\hline 11 & 0 & 10 & 20 & 25 & 13 & 68 \\
\hline 12 & 0 & 0 & 14 & 50 & 4 & 68 \\
\hline 13 & 0 & 20 & 32 & 16 & 0 & 68 \\
\hline 14 & 0 & 14 & 25 & 29 & 0 & 68 \\
\hline 15 & 0 & 42 & 13 & 4 & 9 & 68 \\
\hline 16 & 0 & 16 & 21 & 26 & 5 & 68 \\
\hline 17 & 0 & 4 & 26 & 38 & 0 & 68 \\
\hline 18 & 0 & 4 & 43 & 16 & 5 & 68 \\
\hline 19 & 0 & 31 & 12 & 25 & 0 & 68 \\
\hline 20 & 0 & 0 & 22 & 38 & 8 & 68 \\
\hline 21 & 0 & 4 & 29 & 35 & 0 & 68 \\
\hline 22 & 4 & 8 & 4 & 37 & 15 & 68 \\
\hline 23 & 0 & 4 & 34 & 21 & 9 & 68 \\
\hline 24 & 4 & 42 & 12 & 10 & 0 & 68 \\
\hline 25 & 4 & 42 & 12 & 10 & 0 & 68 \\
\hline 26 & 0 & 12 & 36 & 15 & 5 & 68 \\
\hline 27 & 0 & 12 & 20 & 31 & 5 & 68 \\
\hline 28 & 0 & 8 & 41 & 14 & 5 & 68 \\
\hline 29 & 0 & 8 & 13 & 47 & 0 & 68 \\
\hline 30 & 4 & 33 & 23 & 8 & 0 & 68 \\
\hline 31 & 8 & 40 & 20 & 0 & 0 & 68 \\
\hline 32 & 0 & 8 & 25 & 30 & 5 & 68 \\
\hline 33 & 0 & 5 & 41 & 22 & 0 & 68 \\
\hline 34 & 4 & 13 & 24 & 17 & 10 & 68 \\
\hline 35 & 0 & 13 & 46 & 9 & 0 & 68 \\
\hline 36 & 17 & 41 & 0 & 10 & 0 & 68 \\
\hline 37 & 4 & 17 & 43 & 4 & 0 & 68 \\
\hline 38 & 0 & 4 & 4 & 13 & 47 & 68 \\
\hline 39 & 0 & 0 & 42 & 9 & 17 & 68 \\
\hline Total & 61 & 613 & 907 & 764 & 307 & 2652 \\
\hline
\end{tabular}


Table 3: Characteristics of study participants $(\mathrm{N}=68)$

\begin{tabular}{lrr}
\hline & Frequency & Percent \\
\hline Sex & & \\
$\quad$ male & 17 & 25.00 \\
female & 51 & 75.00 \\
Age & & \\
$<=65$ y/o & 23 & 33.82 \\
$65-75$ y/o & 32 & 47.06 \\
$>75$ y/o & 13 & 19.12 \\
Presence of body functional limitations & & 52.94 \\
Visual defect & 36 & 14.71 \\
Hearing defect & 10 & 45.59 \\
Arthritis disease & 31 & \\
\hline
\end{tabular}

\subsection{Multivariate analysis}

The final result of ordinal logistic regression analysis showed individual demographical, body functional and street built environmental variables fitted the model well and the regression model was valid $\left(-2\right.$ Likelihood ratio chi-square $=1108.16, d f=37$, Nagelkerke pseudo- $\left.R^{2}=0.364, p<0.001\right)$. The regression model results were listed in Table 4. Neither gender (being male, $\beta=-0.116, p=0.200$ ) nor age difference (65-75 y/o, $\beta=0.157, p=0.059 ;>75 \mathrm{y} / \mathrm{o}, \beta=0.173, p=0.105)$ was observed to be significantly correlated with older pedestrians' perceived safety. The presence of functionality limitations, like arthritis disease $(\beta=-0.179, p=0.023)$, showed a negative impact on the perceived safety among the older pedestrians, whereas visual impairment $(\beta=0.128, p=0.091)$ and hearing impairments $(\beta=-0.181, p=0.090)$ were not significant in the regression model.

Significant associations were also observed between older pedestrians' perceived safety and several streetscape characteristics, after adjusting for individual demographic and functional limitation factors. In interface zones, the presence of walls $(\beta=-1.085, p<0.001)$ and commercial activities $(\beta=1.159$, $p=0.006$ ) were significantly negatively and positively associated with an increased perceived safety level, compared to the street interface composed by fences, respectively. Negative associations were also observed when outdoor seats $(\beta=-1.194, p=0.013)$, an elevation difference of 3 -steps $(\beta=-3.115, p<0.001)$ and 10-steps $(\beta=-4.406, p<0.001)$ were presented. Variable in sidewalk zones like footpath widths at 3.0 meters $(\beta=0.703, p<0.001)$ was positively correlated with a higher safety assessment compared to those with 1.5-meter widths. With an increment of crowdedness of the street, older pedestrians' perceived safety decreased, though significances were not steadily shown across all crowdedness levels. The presence of poor maintenance of sidewalk was slightly negatively correlated with increased perceived safety $(\beta=-0.410, p=0.052)$. The presence of tactile paving facilities $(\beta=-0.361, p=0.031)$ also showed an inhibiting effect on a higher level of safety assessment. Compared to quadrate paver textures, pavement surfaced with parallel textures $(\beta=-0.785, p<0.001)$ had a negative correlation with increased perceived safety of older pedestrian. In isolation zones, both the absence of isolation facilities $(\beta=-0.784, p<0.001)$ and presence of bushes $(\beta=-2.666, p<0.001)$ had negative impacts whereas handrails $(\beta=1.566, p<0.001)$ and trees $(\beta=1.800, p<0.001)$ had positive impacts on a higher level of perceived safety, compared to the mixed type of isolation strips. In vehicle zones, both the presence of parking lots $(\beta=-0.642, p<0.001)$ and non-motor vehicle $(\beta=-1.071, p<0.001)$ were negatively correlated with an increased safety percep- 
tion. The presence of medium vehicle volumes $(\beta=0.980, p<0.001)$, compared to that of high traffic volumes, were positively correlated with perceived safety related to walking.

Table 4. Results of ordinal logit regression analysis, including individual demographical variables, body functional limitation variables and street characteristic variables

\begin{tabular}{|c|c|c|c|c|c|c|}
\hline & $\beta$ & S.E & t-value & p-value & \multicolumn{2}{|c|}{ [95\% Confidence Interval] } \\
\hline Sex(male) & -0.116 & 0.090 & -1.28 & 0.200 & -0.292 & 0.061 \\
\hline \multicolumn{7}{|l|}{ Age } \\
\hline $65-75 y / o$ & 0.157 & 0.083 & 1.89 & 0.059 & -0.006 & 0.320 \\
\hline$>=75 \mathrm{y} / \mathrm{o}$ & 0.173 & 0.106 & 1.62 & 0.105 & -0.036 & 0.381 \\
\hline Vision defect & 0.128 & 0.076 & 1.69 & 0.091 & -0.021 & 0.277 \\
\hline Hearing defect & -0.181 & 0.107 & -1.70 & 0.090 & -0.391 & 0.028 \\
\hline Arthritis disease & -0.179 & 0.079 & -2.27 & 0.023 & -0.333 & -0.024 \\
\hline \multicolumn{7}{|l|}{ Interface type } \\
\hline Wall & -1.085 & 0.228 & -4.77 & 0.000 & -1.531 & -0.639 \\
\hline Commercials & 1.159 & 0.422 & 2.75 & 0.006 & 0.332 & 1.986 \\
\hline Plantation & 0.242 & 0.237 & 1.02 & 0.306 & -0.221 & 0.706 \\
\hline Outer settings & -1.194 & 0.480 & -2.49 & 0.013 & -2.134 & -0.253 \\
\hline Steps & & . & . & $\cdot$ & . & • \\
\hline 3-step & -3.115 & 0.444 & -7.02 & 0.000 & -3.985 & -2.245 \\
\hline 10-step & -4.406 & 0.378 & -11.65 & 0.000 & -5.147 & -3.665 \\
\hline \multicolumn{7}{|l|}{ Footpath width } \\
\hline 3.0 meter & 0.703 & 0.195 & 3.60 & 0.000 & 0.320 & 1.086 \\
\hline 5.0 meter & 0.313 & 0.251 & 1.25 & 0.212 & -0.179 & 0.805 \\
\hline \multicolumn{7}{|l|}{ Crowdedness } \\
\hline 2 & -2.323 & 0.651 & -3.57 & 0.000 & -3.600 & -1.046 \\
\hline 3 & -0.722 & 0.465 & -1.55 & 0.120 & -1.633 & 0.188 \\
\hline 4 & -1.394 & 0.542 & -2.57 & 0.010 & -2.457 & -0.331 \\
\hline 5 & -0.802 & 0.446 & -1.80 & 0.072 & -1.676 & 0.073 \\
\hline 6 & -0.659 & 0.514 & -1.28 & 0.199 & -1.666 & 0.348 \\
\hline 7 & -2.641 & 0.621 & -4.25 & 0.000 & -3.857 & -1.424 \\
\hline 8 & -2.144 & 0.428 & -5.01 & 0.000 & -2.983 & -1.306 \\
\hline 9 & -0.541 & 0.394 & -1.37 & 0.170 & -1.313 & 0.232 \\
\hline Maintenance(poor) & -0.410 & 0.211 & -1.94 & 0.052 & -0.824 & 0.004 \\
\hline Tactile paving & -0.361 & 0.167 & -2.16 & 0.031 & -0.688 & -0.034 \\
\hline Pavers directions & 0.000 & & . & . & . & . \\
\hline Parallel & -0.785 & 0.208 & -3.77 & 0.000 & -1.192 & -0.377 \\
\hline Vertical & -0.211 & 0.220 & -0.96 & 0.335 & -0.642 & 0.219 \\
\hline \multicolumn{7}{|l|}{ Isolation type } \\
\hline None & -0.784 & 0.228 & -3.44 & 0.001 & -1.230 & -0.338 \\
\hline Handrail & 1.566 & 0.222 & 7.06 & 0.000 & 1.131 & 2.001 \\
\hline Flower bed & -0.179 & 0.220 & -0.81 & 0.416 & -0.611 & 0.253 \\
\hline Pile & 0.339 & 0.321 & 1.06 & 0.291 & -0.290 & 0.968 \\
\hline Bicycle dock & 0.766 & 0.415 & 1.85 & 0.065 & -0.048 & 1.580 \\
\hline Bushes & -2.666 & 0.360 & -7.41 & 0.000 & -3.371 & -1.960 \\
\hline Trees & 1.800 & 0.349 & 5.15 & 0.000 & 1.115 & 2.485 \\
\hline
\end{tabular}




\begin{tabular}{lrrrrrr}
\hline & $\boldsymbol{\beta}$ & $\boldsymbol{S . E}$ & $\boldsymbol{t}$-value & $\boldsymbol{p}$-value & [95\% Confidence Interval] \\
\hline Parking lot & -0.642 & 0.167 & -3.84 & 0.000 & -0.970 & -0.315 \\
Non-motor vehicle & -1.071 & 0.227 & -4.73 & 0.000 & -1.515 & -0.627 \\
Motor vehicle & & & & & & \\
Low & 0.333 & 0.195 & 1.71 & 0.088 & -0.049 & 0.715 \\
Medium & 0.980 & 0.199 & 4.93 & 0.000 & 0.590 & 1.370 \\
\hline
\end{tabular}

Texts in bold indicate the variable feature was significant at 0.05 level $(p<0.05) . \beta$, the coefficient of regression analysis, S.E, standard error. Reference category of each variable was not shown in this table but in Table 1 .

\section{$5 \quad$ Discussion}

Based on 2652 safety ratings on 39 simulated streetscape pictures, the research quantitatively investigated the impact that individual confounders and street built environment characteristics had on safety perception among a small group of older pedestrians in Shanghai, China. Additionally, we conducted a series of in-depth interviews, which aimed to better understand and generalize the quantitative research results.

\subsection{Individual-level factors}

The roles of gender and age differences in the walking safety assessment were not explicit in the current research, such a finding was inconsistent with previous research which found a higher level of perceived safety from males' perspective (Dawson, Hillsdon, Boller, \& Foster, 2007). Significant impacts of age and gender were absent due partly to the small sample size and the disproportionate gender distribution. Instead, functionality limitation among elders, like arthritis disease, was found negatively related to individual perceived safety. As previous clinical research on gait changes of older adults indicated, the decreased lower-extremity capacity was associated with fear of falling (Maki, 1997). This was also verified in the in-depth interview where two participants reported they had severe arthritis diseases thus leading one participant to be more static at home. The significant impact of vision impairments on perceived safety was not found in the quantitative analysis but in the in-depth interviews, several participants reported that they had suffered severe visual declines as they aged, and reported they had to be more cautious when walking outside. Based on the quantitative and qualitative analysis results, the researchers inferred that the adverse impacts brought by mobility decline issues (such as arthritis disease) were more severe than those by a sensory decline (visual or hearing). However, the details beneath the inference need to be further investigated using a larger sample.

"As aged, my physical capacities declined drastically. I got anchyloses which prevent me from outdoor activities."-female, age 65

"I had poor vision. So I cannot be too cautious when I walk out. "-female, age 63

\subsection{Building-street interface}

The adverse impacts that walls in street interface zone had on older pedestrians' safety perception were mainly due to the feelings of suppression created by bald walls. According to environmental psychol- 
ogy theory, perceived safety would drastically decrease due to the fear of crime or the strangers in a constrained space (Ashihara, 1981). To the contrary, the commercial activities along the street, which were often used as proxies of urban vitality (Ye et al., 2018), were conducive to a higher level of perceived safety because of the lively scenes, which reduced the fear of crime of older pedestrians (Foster et al., 2010). Though a relatively lower crime incident rate was reported in most Chinese cities, the issue of older pedestrians' perceived safety from crime on streets still needs to be emphasized because of the decreased reaction speeds and flexibility among older pedestrians, especially when one is suffering from functionality limitations. To be noted, the arrangement of street commercial activities should also be temperate, as the negative effects of outer setting (i.e., outdoor seats) was also found in the current study, which could be seen as an overrun, impeding the pedestrian flow thus led to a decreased perceived safety among older pedestrians. On the other hand, this negative impact complied with the walking demand theory which inferred that although the presence of street seating was a crucial indicator of a high quality and comfortable walkable environment, individual would not engage in walking activities if their lower-level need such as safety was not met (Alfonzo, 2005). The voices of interviewees also proved this finding. Interviewees preferred to street settings with more urban furniture, meanwhile they tolerated the flaws of the existing environment. Compared to a flat sidewalk base, the presence of steps in sidewalks brought about a decreased level of safety perception because the elevation difference introduced a more complicated walking environment for the elderly. Given the decreased lower-extremity capacity and gait stride changed with people age (Lu, Chen, \& Chen, 2006), older pedestrians had to cope with greater challenges of negotiating with steps on the sidewalk. A slight slope was recommended in previous research on inclusive street design for seniors with mobility limitations (Burton \& Mitchell, 2006).

\section{"It couldn't get better if enough seats were just placed along the road."-female, age 74}

"If I feel tired, I'll definitely stop and find a place to sit down regardless of tidiness."-male, age 70

\subsection{Sidewalk}

Although the pedestrian crowds indicated the livability of the street (Gehl, 2013; Jacobs, 1993), a higher level of pedestrian traffic led to a higher risk of collision or fall in overcrowded situations, negatively impacting perceived safety of older pedestrians. Crowdedness was a comprehensive factor which took footpath width, sidewalk obstacles, pedestrian flow and many other variables into account. In the in-depth interview, several participants complained of the inappropriate behaviors, such as invasion of street by personal goods, which often led to an inadequacy of available walking space on sidewalks in their daily lives. Older pedestrians were more sensitive to narrow street spaces as a previous clinical research found older adults who were constrained to the age-related hip joint functional declines had to rotate their shoulders more than the youngers do when passing through narrow spaces safely (Hackney \& Cinelli, 2011). Inclusive street design principles also suggested a safe sidewalk should at least be as wide as 2 meters, to provide a safe place to travel, especially for pedestrians who suffered from mobility limitations (Burton \& Mitchell, 2006). However, when the footpath width increased to 5.0 meters in the current research, the significant association between footpath width and perceived safety vanished. Again, this is another story related to the perceived comfort rather than safety issue in a walking environment.

The negative correlation between presence of pavement surfaced with parallel textures and older pedestrians' perceived safety was observed. Compared to the sidewalk surfaced with quadrate paver textures, parallel tile textures magnified the perspective effects of the pavers which might lead to a distraction during walking, especially for those with visual impairments. This finding was consistent with a 
previous qualitative study stating that complicated pavement patterns created confusion for older adults with visual impairments (Burton \& Mitchell, 2006).

"The sidewalk should be as wide as 3.0 meter but now it was invaded by illegal constructs."-male, age 80

"The footpath is too narrow due to the invasions of commercial signage and personal staffs"-female, age 63

The demand of sidewalks with even surfaces was reported by interviewees. As indicated in many previous research projects (Barnett et al., 2016; Clarke, Ailshire, Bader, Morenoff, \& House, 2008; Joseph \& Zimring, 2007), the term "evenness" was a crucial factor in older adult walking activities. Compared to younger pedestrians who preferred a shortcut or the fastest passage, older pedestrians focused more on the presence and quality of sidewalk on their routes (Bernhoft \& Carstensen, 2008). The heavy demand on evenness also interpreted the negative association between presence of tactile paving and perceived safety in the quantitative analysis. Due to age-related decreased body somatosensory function, increased postural sway was observed in older people, which increased the possibility of fall of older adults (Qiu et al., 2012). Although tactile paving is an essential amenity in an inclusive urban environment, its raised surface, typically consisted of tough bumps, was a deterrent to older adults who did not suffer from severe visual impairment.

"We never excessively demand any luxury pavement. Pavement being even is the biggest thing."

-male, age 70

"The surface must be even. We don't like any bumps."-female, age 74

"The rough pavement surface were prevalent in surrounding areas. It causes many disgusting puddles on rainy days." -male, age 70

\subsection{Isolation facilities}

The isolation strip provided a physical barrier between pedestrian and non-motor vehicle traffic. Different types of isolation facilities, due to their unique attributes such as width, height, and continuity, introduced various safety perceptions among older pedestrians. The presence of bushes blocked both pedestrians' and vehicle drivers' visions and increased the possibilities of collision. On the contrary, the presence of handrails was found positively correlated with perceived safety because of its continuity and vision permeability. The positive effects of street trees on increased perceived safety were found in the current research and also testified by a recent research on elderly pedestrian collisions which showed the higher the ratio of the intersection areas covered by street trees and their shade, the less likely an older pedestrian would be involved in collision (Kim, 2019). Another research also indicated that the presence of street trees enhanced the streetscape enclosure and the tree canopy created room-like spaces where pedestrians would perceive safer than other street settings (Harvey et al., 2015). According to a previous research on pedestrians' perceptions of local street service (Kang et al., 2013), non-motor vehicle facility on the sidewalk, such as bike dock, was expected to be negatively correlated with increased perceived safety. Besides, the event of picking up a bicycle from dock would interrupt pedestrian flow on the sidewalk. However, no significant association was found in the current quantitative analysis. In the in-depth 
interview statements, some participants reported their fear of collision with bicycles on the sidewalk. The impact of sidewalk bicycle riders on older pedestrians' perceived safety needs further investigation in the future.

\section{"Sometimes delivery men riding e-bicycles break the rules, leading to collisions with pedestrians." -male, age 80}

"Now cars are parked everywhere. I had to switch to the vehicle lane because the parked car obstructed my way."-female, age 74

\subsection{Vehicle traffic}

Both the presence of off-street car parking and bike riders were negatively correlated with the perceived safety of older pedestrians. Issues of e-bicycle have received wider attentions than ever before in China, as the traffic incidents involved with e-bikes has led to thousands of injuries and fatalities in recent years. Many interviewees reported that non-motor vehicles (e-bicycle) were the most serious threat to their walking activities. Some interviewees also complained about the illegal car parking on the sidewalk and that along the road. They stated they had to change their routes and yielded to the inappropriately parked cars on the sidewalk. It also indicated the contradiction between people's demand for private cars and the insufficiency of urban parking facilities, which aggravated with urban economic development in Chinese cities. Compared to a high vehicle traffic volume, a moderate volume of vehicle traffic increased older pedestrians' perceived safety. This is because, to some extent, a moderate volume of cars reflected the livability of streets, which was proved by previous researches that elderly people were more likely to walk along main street where a higher level of traffic presented (Borst et al., 2009), considering the attractiveness of busy streets (i.e., human activity) (Borst, Miedema, de Vries, Graham, \& van Dongen, 2008). However, we also need to pay attention to the traffic burdens to older pedestrians. As people age, the decreased processing speed and visual attention abilities resulted in more incorrect decisions when dealing with traffics (Dommes \& Cavallo, 2011). Only by optimizing the facilities and right-of-way will we realize a balanced, sustainable street which facilitates multiple users, supporting sustainable travel modes as well as maintaining a safe walkable environment for older adults.

"Pedestrians walk on the sidewalk, bicycle riders also ride on the sidewalk, that will result in the collision." -male, age 63

"The riders on the sidewalk make me feel unsafe and fearful."-male, age 70

\section{Conclusion}

The research employed a mixed research approach to explore the relationship between older pedestrians' perceived safety and street built environmental characteristics meanwhile adjusting for individual factors like gender, age, and functional limitations. Using the simulated streetscape pictures of most parsimony, a novel survey protocol was developed to measure older pedestrians' perceived safety. Several key attributes of street characteristics, like interface type, elevation differences, footpath width, isolation facility type, as well as traffic issues were found to be related to older pedestrians' perceived safety. One strength of the research is the development of a protocol using simulated streetscape images. The simu- 
lated streetscapes could minimize the impacts of exogenous confounders like weather conditions and air quality on the walking safety assessment. Another strength of this study was the depth of insight into research subjects. We investigated individual-level attributes and found the magnitudes of functional limitations were more crucial than those of age and gender differences in the current older pedestrians' perceived safety study. This indicated, when dealing with issues of built environment and older adults' travel behaviors, greater attentions should be paid to those who suffered from age-related functional declines. The results of the research can help guide inclusive urban street design and transportation management and planning both in China and other highly-populated areas.

This research still has limitations. Some research results of this cross-sectional study may be biased due to the small sample size and gender disproportion. The voluntarily recruitment process could guarantee the quality of survey from individual level, whereas generating a biased gender distribution which weakened the generalizability of the results. While the simulated streetscape images could minimize the interferential factors in the real world, other real-time street characteristic such as bicycle volume was difficult to present due to the limitation of static pictures. As aging is a process, longitudinal studies and time-series data are suggested in future researches on older pedestrians' activities, especially for the cohort with extensive functionality limitations related to walking, such as cognitive or visual declines. Novel streetscape survey protocol like video clips and effective measurement approach like online survey and big data should also be considered in future research related to pedestrians and safety.

\section{Acknowledgments}

This research was supported by The National Natural Science Foundation of China (No. 51278339 and No. 51678414), the China Scholarship Council (No. 201706260167), and USDOT the Consortium of Cooperative Mobility for Competitive Megaregions Center. The authors acknowledge Yanli Ma for data collection assistance, Chris Bischak for language revision, and all anonymous reviewers for their valuable comments and guidance.

\section{Data availability}

The data used in this research is self-archived and available in:

https://drive.google.com/file/d/19lIdYs-W5VhnAVfrPUnz9jFTM1ZGvc6f/view?usp=sharing

Authors highly suggest future user(s) contact corresponding author prior to any reproduction. 


\section{References}

Alfonzo, M. A. (2005). To walk or not to walk? The hierarchy of walking needs. Environment and Behavior, 37(6), 808-836. https://doi.org/10.1177/0013916504274016

Ashihara, Y. (1981). Exterior design in architecture. New York: Van Nostrand Reinhold Company.

Barnett, A., Cerin, E., Zhang, C. J. P., Sit, C. H. P., Johnston, J. M., Cheung, M. M. C., \& Lee, R. S. Y. (2016). Associations between the neighborhood environment characteristics and physical activity in older adults with specific types of chronic conditions: The ALECS cross-sectional study. International Journal of Behavioral Nutrition and Physical Activity, 13, 53. https://doi.org/10.1186/ s12966-016-0377-7

Bernhoft, I. M., \& Carstensen, G. (2008). Preferences and behavior of pedestrians and cyclists by age and gender. Transportation Research Part F: Traffic Psychology and Behavior, 11(2), 83-95. https://doi. org/10.1016/j.trf.2007.08.004

Booth, M. L., Owen, N., Bauman, A., Clavisi, O., \& Leslie, E. (2000). Social-cognitive and perceived environment influences associated with physical activity in older Australians. Preventive Medicine, 31(1), 15-22. https://doi.org/10.1006/pmed.2000.0661

Borst, H. C., de Vries, S. I., Graham, J. M. A., van Dongen, J. E. F., Bakker, I., \& Miedema, H. M. E. (2009). Influence of environmental street characteristics on walking route choice of elderly people. Journal of Environmental Psychology, 29(4), 477-484. https://doi.org/10.1016/j.jenvp.2009.08.002

Borst, H. C., Miedema, H. M. E., de Vries, S. I., Graham, J. M. A., \& van Dongen, J. E. F. (2008). Relationships between street characteristics and perceived attractiveness for walking reported by elderly people. Journal of Environmental Psychology, 28(4), 353-361. https://doi.org/10.1016/j.jenvp.2008.02.010

Bureau of Statistics of Shanghai. (2018). Shanghai statistical yearbook 2018. Retrieved from http://tjj. sh.gov.cn/html/sjfb/201901/1003014.html

Burton, E., \& Mitchell, L. (2006). Inclusive urban design: Streets for life. Abingdon, UK: Routledge.

Cao, X., Mokhtarian, P. L., \& Handy, S. L. (2010). Neighborhood design and the accessibility of the elderly: An empirical analysis in Northern California. International Journal of Sustainable Transportation, 4(6), 347-371. https://doi.org/10.1080/15568310903145212

Chaudhury, H., Campo, M., Michael, Y., \& Mahmood, A. (2016). Neighborhood environment and physical activity in older adults. Social Science \& Medicine, 149, 104-113. https://doi.org/10.1016/j. socscimed.2015.12.011

Chen, H., Jia, B., \& Lau, S. S. Y. (2008). Sustainable urban form for Chinese compact cities: Challenges of a rapid urbanized economy. Habitat International, 32(1), 28-40. https://doi.org/10.1016/j.habitatint.2007.06.005

Chen, W. (2010). Age differences in community participation of urban residents (in Chinese). Journal of Jianghan University, 27(4), 84-89. https://doi.org/10.16387/j.cnki.42-1867/c.2010.04.006

Chen, Y., Jiao, J., Mao, J., \& Wu, H. (2017). Understanding pedestrians' travel behavior in large Chinese cities: A case study of Shanghai central city. Transportation Research Procedia, 25, 2287-2296. https://doi.org/10.1016/j.trpro.2017.05.440

Clarke, P., Ailshire, J. A., Bader, M., Morenoff, J. D., \& House, J. S. (2008). Mobility disability and the urban built environment. American Journal of Epidemiology, 168(5), 506-513. https://doi. org/10.1093/aje/kwn185

Dawson, J., Hillsdon, M., Boller, I., \& Foster, C. (2007). Perceived barriers to walking in the neighborhood environment: A survey of middle-aged and older adults. Journal of Aging and Physical Activity, 15(3), 318-335. Retrieved from https://www.ncbi.nlm.nih.gov/pubmed/17724397 
Dommes, A., \& Cavallo, V. (2011). The role of perceptual, cognitive, and motor abilities in street-crossing decisions of young and older pedestrians. Ophthalmic and Physiological Optics, 31(3), 292-301. https://doi.org/10.1111/j.1475-1313.2011.00835.x

Ewing, R., \& Handy, S. (2009). Measuring the unmeasurable: Urban design qualities related to walkability. Journal of Urban Design, 14(1), 65-84. https://doi.org/10.1080/13574800802451155

Feng, J. (2017). The influence of built environment on travel behavior of the elderly in urban China. Transportation Research Part D: Transport and Environment, 52, 619-633. https://doi.org/10.1016/j. $\operatorname{trd} .2016 .11 .003$

Foster, S., Giles-Corti, B., \& Knuiman, M. (2010). Neighborhood design and fear of crime: A socialecological examination of the correlates of residents' fear in new suburban housing developments. Health Place, 16(6), 1156-1165. https://doi.org/10.1016/j.healthplace.2010.07.007

Foster, S., Hooper, P., Knuiman, M., Christian, H., Bull, F., \& Giles-Corti, B. (2016). Safe RESIDential environments? A longitudinal analysis of the influence of crime-related safety on walking. International Journal of Behavioral Nutrition and Physical Activity, 13(1), 22. https://doi.org/10.1186/ s12966-016-0343-4

Frank, L. D., Sallis, J. F., Saelens, B. E., Leary, L., Cain, K., Conway, T. L., \& Hess, P. M. (2010). The development of a walkability index: Application to the neighborhood quality of life study. British Journal of Sports Medicine, 44(13), 924-933. https://doi.org/10.1136/bjsm.2009.058701

Gehl, J. (2013). Cities for people. Washington, DC: Island Press.

Gibson, J. J. (1977). The theory of affordances. In The ecological approach to visual perception (pp. 127137). Hillsdale, NJ: Lawrence Erlbaum Associates.

Hackney, A. L., \& Cinelli, M. E. (2011). Action strategies of older adults walking through apertures. Gait \& Posture, 33(4), 733-736.

Harvey, C., Aultman-Hall, L., Hurley, S. E., \& Troy, A. (2015). Effects of skeletal streetscape design on perceived safety. Landscape and Urban Planning, 142, 18-28. https://doi.org/10.1016/j.landurbplan.2015.05.007

Jacobs, A. B. (1993). Great streets. Cambridge, MA: MIT Press.

Jacobs, J. (1961). The death and life of great American cities. New York: Random House.

Joseph, A., \& Zimring, C. (2007). Where active older adults walk: Understanding the factors related to path choice for walking among active retirement community residents. Environment and Behavior, 39(1), 75-105. https://doi.org/10.1177/0013916506295572

Kang, L., Xiong, Y., \& Mannering, F. L. (2013). Statistical analysis of pedestrian perceptions of sidewalk level of service in the presence of bicycles. Transportation Research Part A: Policy and Practice, 53, 10-21. https://doi.org/10.1016/j.tra.2013.05.002

Kim, D. (2019). The transportation safety of elderly pedestrians: Modeling contributing factors to elderly pedestrian collisions. Accident Analysis \& Prevention, 131, 268-274. https://doi.org/10.1016/j. aap.2019.07.009

Li, F., Fisher, J., \& Brownson, R. C. (2005). A multilevel analysis of change in neighborhood walking activity in older adults. Journal of Aging and Physical Activity, 13(2), 145-159. Retrieved from https://www.ncbi.nlm.nih.gov/pubmed/15995261

Li, X., Zhang, C., Li, W., Ricard, R., Meng, Q., \& Zhang, W. (2015). Assessing street-level urban greenery using Google street view and a modified green view index. Urban Forestry \& Urban Greening, 14(3), 675-685. https://doi.org/10.1016/j.ufug.2015.06.006

Loukaitou-Sideris, A. (2006). Is it safe to walk? Neighborhood safety and security considerations and their effects on walking. Journal of Planning Literature, 20(3), 219-232. https://doi. org/10.1177/0885412205282770

Lu, T.-W., Chen, H.-L., \& Chen, S.-C. (2006). Comparisons of the lower limb kinematics between young and older adults when crossing obstacles of different heights. Gait \& Posture, 23(4), 471-479. 
Maki, B. E. (1997). Gait changes in older adults: Predictors of falls or indicators of fear. Journal of the American Geriatrics Society, 45(3), 313-320. Retrieved from https://www.ncbi.nlm.nih.gov/ pubmed/9063277

Marshall, W. E., \& Garrick, N. W. (2011). Does street network design affect traffic safety? Accident Analysis \& Prevention, 43(3), 769-781. https://doi.org/10.1016/j.aap.2010.10.024

Maslow, A. H. (1943). A theory of human motivation. Psychological Review, 50(4), 370.

Michael, Y., Beard, T., Choi, D., Farquhar, S., \& Carlson, N. (2006). Measuring the influence of built neighborhood environments on walking in older adults. Journal of Aging and Physical Activity, 14(3), 302-312. Retrieved from https://www.ncbi.nlm.nih.gov/pubmed/17090807

National Bureau of Statistics of China. (2019). Statistics of year. Retrieved from http://data.stats.gov.cn/ easyquery.htm?cn=C01\&zb=A0301\&sj=2018

Pikora, T., Giles-Corti, B., Bull, F., Jamrozik, K., \& Donovan, R. (2003). Developing a framework for assessment of the environmental determinants of walking and cycling. Social Science \& Medicine, 56(8), 1693-1703. https://doi.org/10.1016/s0277-9536(02)00163-6

Qiu, F., Cole, M. H., Davids, K., Hennig, E., Silburn, P., Netscher, H., \& Kerr, G. (2012). Enhanced somatosensory information decreases postural sway in older people. Gait \& Posture, 35(4), 630-635.

Ross, P. J. (1988). Taguchi techniques for quality engineering: Loss function, orthogonal experiments, parameter and tolerance design. Toronto: McGraw-Hill.

Sallis, J. F., Cervero, R. B., Ascher, W., Henderson, K. A., Kraft, M. K., \& Kerr, J. (2006). An ecological approach to creating active living communities. Annual Review of Public Health, 27, 297-322. https://doi.org/10.1146/annurev.publhealth.27.021405.102100

Schlozman, K. L., Burns, N., Verba, S., \& Donahue, J. (1995). Gender and citizen participation: Is there a different voice? American Journal of Political Science, 39(2), 267-293. https://doi. org/10.2307/2111613

Shumake, K. L., Sacks, J. D., Lee, J. S., \& Johns, D. O. (2013). Susceptibility of older adults to health effects induced by ambient air pollutants regulated by the European Union and the United States. Aging Clinical and Experimental Research, 25(1), 3-8. https://doi.org/10.1007/s40520-013-0001-5

Tana, M.-P. K., \& Chai, Y. (2015). Urban form, car ownership and activity space in inner suburbs: A comparison between Beijing (China) and Chicago (United States). Urban Studies, 53(9), 1784 1802. https://doi.org/10.1177/0042098015581123

Traunmueller, M., Marshall, P., \& Capra, L. (2016). ... When you're a stranger: Evaluating safety perceptions of (un)familiar urban places. Paper presented at the Proceedings of the Second International Conference on IoT in Urban Space, Tokyo, Japan. Retrieved from https://dl.acm.org/ doi/10.1145/2962735.2962761

Tulloch, M. (2000). The meaning of age differences in the fear of crime. British Journal of Criminology, $40(3), 451-467$.

Van Cauwenberg, J., Van Holle, V., De Bourdeaudhuij, I., Clarys, P., Nasar, J., Salmon, J., . . Deforche, B. (2014). Physical environmental factors that invite older adults to walk for transportation. Journal of Environmental Psychology, 38, 94-103. https://doi.org/10.1016/j.jenvp.2013.12.012

Wardman, M. (1988). A comparison of revealed preference and stated preference models of travel behavior. Journal of Transport Economics and Policy, 71-91.

Wu, H., Chen, Y., \& Jiao, J. (2019). Impact of neighborhood built environments on shopping travel modes in Shanghai, China. Transportation Research Record: Journal of the Transportation Research Board, 2673(8), 669-681. https://doi.org/10.1177/0361198119844969

Yang, J., \& Gakenheimer, R. (2007). Assessing the transportation consequences of land-use transformation in urban China. Habitat International, 31(3-4), 345-353. https://doi.org/10.1016/j.habitatint.2007.05.001 
Ye, Y., Li, D., \& Liu, X. (2018). How block density and typology affect urban vitality: An exploratory analysis in Shenzhen, China. Urban Geography, 39(4), 631-652. https://doi.org/10.1080/0272363 8.2017 .1381536

Ye, Y., Richards, D., Lu, Y., Song, X., Zhuang, Y., Zeng, W., \& Zhong, T. (2019). Measuring daily accessed street greenery: A human-scale approach for informing better urban planning practices. Landscape and Urban Planning, 191,103434.

Zamora, T., Alcántara, E., Artacho, M. Á., \& Cloquell, V. (2008). Influence of pavement design parameters in safety perception in the elderly. International Journal of Industrial Ergonomics, 38(11-12), 992-998.

Zhang, X., Xiang, H., Jing, R., \& Tu, Z. (2011). Road traffic injuries in the People's Republic of China, 1951-2008. Traffic Injury Prevention, 12(6), 614-620. https://doi.org/10.1080/15389588.2011.6 09925 